\title{
The Vienna Bone and Growth Center - care and research in the field of rare diseases
}

\author{
Gabriele Haeusler · Rudolf Ganger · Roland Kocijan · Nadja Fratzl-Zelman
}

Accepted: 21 January 2021

(C) Springer-Verlag GmbH Austria, ein Teil von Springer Nature 2021

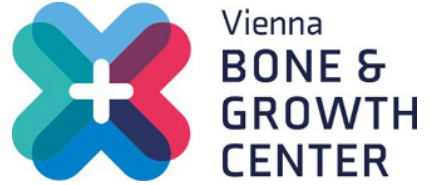

Dear colleagues,

It is our pleasure to present this special issue of the Wiener Medizinische Wochenschrift (WMW) on the occasion of the designation of our center of expertise for rare disorders of bone, mineralization, and growth-the Vienna Bone and Growth Center (VBGC)-by the Austrian Federal Ministry of Social Affairs, Health, Care, and Consumer Protection in April 2020. In a unique collaboration of four spe-

Electronic supplementary material The online version of this article (https://doi.org/10.1007/s10354-021-00822-0) contains supplementary material, which is available to authorized users.

\section{G. Haeusler $(\bowtie)$}

Department of Pediatrics and Adolescent Medicine, Comprehensive Center for Pediatrics, Medical University of Vienna, Waehringer Guertel 18-20, 1090 Vienna, Austria gabriele.haeusler@meduniwien.ac.at

\section{R. Ganger}

Department of Pediatric Orthopaedics, Orthopaedic Hospital Speising, Speisinger Straße 109, 1130 Vienna, Austria

\section{R. Kocijan}

1st Medical Department at Hanusch Hospital, Hanusch Hospital of the OEGK, Heinrich-Collin-Str. 30, 1140 Vienna, Austria

\section{R. Kocijan · N. Fratzl-Zelman}

1st Medical Department Hanusch Hospital, Ludwig Boltzmann Institute of Osteology at the Hanusch Hospital of OEGK and AUVA Trauma, Centre Meidling,

Heinrich-Collin-Str. 30, 1140 Vienna, Austria

G. Haeusler · R. Ganger · R. Kocijan · N. Fratzl-Zelman

Vienna Bone and Growth Center, Vienna, Austria cialized centers in Vienna, a multidisciplinary team within the VBGC cares for patients with these rare disorders from birth to adulthood.

Care for rare disorders has become an important topic within the European Union. European reference networks (ERNs) have been set up with the goal of offering patient care at the highest level in member states. At the same time, ERNs facilitate international expert rounds on individual patients and offer new possibilities in teaching as well as clinical and basic research. Last but not least, ERNs offer support of patient advocacy groups.

The VBGC is structured paninstitutionally, bringing together multidisciplinary teams consisting of clinicians (pediatric and adult endocrinologists, osteologists, clinical geneticists, pediatric and adult orthopedic surgeons, radiologists), basic scientists, and functional therapeutic teams specialized in bone and growth disorders. Detailed information on our teams can also be found on our website www.vbgc.at.

The articles presented in this issue of the WMW provide a selection from the broad spectrum of disorders treated at the VBGC. In the present issue, we give a short overview on the clinical work and the research activities of the Vienna Bone and Growth Center for the interested reader.

We hope that you enjoy these articles.

The coordinators of the Vienna Bone and Growth Center

Conflict of interest G. Haeusler, R. Ganger, R. Kocijan, and $\mathrm{N}$. Fratzl-Zelman declare that they have no competing interests.

Publisher's Note Springer Nature remains neutral with regard to jurisdictional claims in published maps and institutional affiliations. 